\title{
242 PHARMACOLOGIC TUMOR PD-L1 DEPLETION WITH CHLORAMBUCIL TREATS OVARIAN CANCER AND MELANOMAS IN A TUMOR PD-L1-DEPENDENT MANNER AND RENDERS $\alpha$ PD-L1-RESISTANT TUMORS $\alpha$ PD-L1- SENSITIVE
}

Haiyan Bai* , Álvaro Padrón, Yilun Deng, Anand Kornepati, Srikanth Polusani, Suresh Kari, Clare Murray, Myrna Garcia, Ryan Reyes, Niannian Ji, Harshita Gupta, Matthew Hart, Tyler Curiel. University of Texas Health San Antonio, San Antonio, TX, USA

Background Programmed cell death ligand-1 (PD-L1) overexpression in tumor cells inhibits $\mathrm{T}$ cells activity and delivers pathologic intracellular signals that can reduce cancer treatment responses in pre-clinicalmodels. ${ }^{12}$

Methods To reduce tumor intracellular PD-L1-mediated pathology, we performed a drug screen that identified chlorambucil as a tumor cell PD-L1 depletion drug.

Results Chlorambucil depletes basal tumor PD-L1 expression through the ubiquitination proteasome pathway. In the tumor microenvironment, high chlorambucil doses treated orthotopic B16 melanoma and ID8agg ovarian cancer. Chlorambucil treatment efficacy was lost or reduced in PD-L1lo ID8agg and PD-L1KO B16 tumors, corroborated with in vitro data. These data suggest that chlorambucil anti-tumor activity of CAMB requires tumor PD-L1 expression, confirmed in PD-L1KO host challenge with CTRL tumor, which chlorambucil treated effectively. Chlorambucil rendered $\alpha$ PD-L1 resistant CTRL ID8agg and PD-L1lo B16 tumors $\alpha$ PD-L1 sensitive, preliminarily possibly due to tumor STING activation, and associated with enhanced tumor NK cell infiltration and central memory $\mathrm{T}$ cell generation. Chlorambucil also phenocopied genetic PDL1KO by reducing tumor cell mTORC1 signals and stem cell content, ${ }^{3}$ suggesting additional treatment potential.

Conclusions Chlorambucil could be a useful strategy to reprogram tumor PD-L1 signals and boost immune-based therapies especially for anti-PD-L1-resistant tumors.

\section{REFERENCES}

1.. Clark CA et al. Tumor-intrinsic PD-L1 signals regulate cell growth, pathogenesis, and autophagy in ovarian cancer and melanoma. Cancer Res 2016;76:69646974.

2.. Juneja VR et al. PD-L1 on tumor cells is sufficient for immune evasion in immunogenic tumors and inhibits CD8 T cell cytotoxicity. J Exp Med 2017;214:895-904.

3.. Gupta HB et al. Tumor cell-intrinsic PD-L1 promotes tumor-initiating cell generation and functions in melanoma and ovarian cancer. Signal Transduct Target Ther 2016;1:2095-9907.

Ethics Approval We received approval from the UT Health San Antonio Institutional Animal Care and Use Committee (IACUC) for each procedure that used mice. We conducted each experiment per the standards required by the UT Health San Antonio Department of Laboratory Animal Resources.

http://dx.doi.org/10.1136/jitc-2021-SITC2021.242 